\title{
Desenvolvimento de uma versão exploratória do Questionário de Atitudes em Relação à População em Situação de Sem-Abrigo: Estudo da estrutura fatorial e características psicométricas
}

\section{Development of an exploratory version of the Attitudes Towards People in a Homeless Situation Questionnaire: Factor structure and psychometric properties study}

\author{
Andreia Cardoso (1) \\ Ana Galhardo (1,2) \\ Ilda Massano-Cardoso $(1,3,4)$ \\ Marina Cunha $(1,2)$
}

(1) Inst Superior Miguel Torga, Portugal

(2) Univ Coimbra, Fac de Psicologia e Ciências da Educação; Centro de Investigação em Neuropsicologia e Intervenção Cognitiva e Comportamental (CINEICC), Portugal

(3) Univ Coimbra, Fac de Medicina, Portugal

(4) Univ Coimbra, Fac de Economia, Centro de Estudos e Investigação em Saúde (CEISUC), Portugal

Recebido: 12/04/2021; Revisto: 04/05/2021; Aceite: 15/05/2021.

\section{Resumo}

Objetivo: Face à escassez de medidas breves que contemplem as três componentes atitudinais (cognitiva, comportamental e emocional) em relação à população em situação de sem-abrigo, o presente estudo teve como objetivo nuclear o desenvolvimento e estudo da versão exploratória de um questionário de atitudes relativamente a esta população. Métodos: A amostra foi constituída por 361 participantes da população geral. Os participantes responderam online a um conjunto de instrumentos de autorresposta, incluindo o Questionário de Atitudes em Relação à População em Situação de Sem-Abrigo (QARPSSA). Resultados: Após a análise fatorial exploratória do QARPSSA, foram excluídos 15 itens, o que resultou numa versão final de 17 itens. O modelo de análise fatorial confirmatória demonstrou uma boa qualidade de ajustamento: $C F I=0,90 ; G F I=0,92 ; R M S E A=0,07$ e $M E C V I=1,02$. Apenas o índice de ajustamento do qui-quadrado normalizado apresentou um valor sofrível $\left(\chi^{2} / g l=2,51\right)$. A variância extraída média foi de 0,49 , valor superior ao quadrado das correlações dos fatores que variou entre 0,002 e 0,25 , sendo sugestivo de validade discriminante. A análise da fidedignidade, através do cálculo do alfa de Cronbach, revelou um valor de 0,77 e através do cálculo da fiabilidade compósita de 0,91. Conclusões: Esta versão exploratória do QARPSSA revelou ser válida e fidedigna para a avaliação das atitudes em relação à população em situação de sem-abrigo, podendo ser usada em vários contextos.

Palavras-Chave: Questionário de Atitudes em Relação à População em Situação de Sem-Abrigo; Atitudes; Sem-abrigo; Estrutura fatorial; Características psicométricas.

\section{DI\&D | ISMT}

rpics@ismt.pt

https://rpics.ismt.pt
Publicação em Acesso Aberto

(C)2021. O(s) Autor(es). Este é um artigo de acesso aberto distribuído sob a Licença Creative Commons Attribution, que permite uso, distribuição e reprodução sem restrições em qualquer meio, desde que o trabalho original seja devidamente citado.
Ana Galhardo

Instituto Superior Miguel Torga,

Largo da Cruz de Celas, n¹, 3000-132

Coimbra, Portugal

E-mail: anagalhardo@ismt.pt 


\begin{abstract}
Objective: Short self-report measures contemplating the three attitudinal components (cognitive, behavioural, and emotional) towards the homeless population are scarce. The current study aimed to develop and study an exploratory version of a new questionnaire targeting attitudes towards homeless people. Method: The sample comprised 361 participants from the general population. Participants completed five self-report instruments, including the Questionnaire on Attitudes Toward Homeless People (QARPSSA). Results: Exploratory factor analysis of the QARPSSA led to the removal of 15 items resulting in a 17-items final version. A confirmatory factor analysis revealed that the three-factor tested model showed a good adjustment quality: $C F I=.90$; $G F I=.92 ; R M S E A=.07$ and $M E C V I=1.02$. Only the normalised chi-square adjustment index showed a poor fit to the data $\left(\chi^{2} / d f\right.$ $=2.51$ ). The average extracted variance was .49 , a value higher than the square of the correlations between the factors (.002 to .25), being suggestive of discriminant validity. Reliability analysis revealed a Cronbach's alpha value of .77 and composite reliability of .91. Conclusions: This exploratory version of the QARPSSA proved to be a valid and reliable instrument for assessing attitudes towards the homeless population, which can be used in several contexts.
\end{abstract}

Keywords: Questionnaire on Attitudes towards the Homeless Population; Attitudes; homeless; Factor structure; Psychometric characteristics.

\title{
Introdução
}

A condição de sem-abrigo não inclui somente as pessoas que vivem, literalmente, na rua. Em Portugal, uma pessoa, independentemente da sua nacionalidade, origem racial ou étnica, religião, idade, sexo, orientação sexual, condição socioeconómica e condição de saúde física e mental, é considerada em situação de semabrigo se estiver sem teto - a viver em espaços públicos, abrigo de emergência ou num local precário - ou sem casa, se estiver em alojamento temporário (Resolução do Conselho de Ministros, n.ㅇ 107/2017). De acordo com os dados da Eurocid, a população em situação de sem-abrigo aumentou de forma exponencial - 157\% - entre 2014 e 2018 (OECD, 2020). Ainda, segundo a Estratégia Nacional para a Integração de Pessoas em Situação de Sem-Abrigo (Grupo de Trabalho para a Monitorização e Avaliação da ENIPSSA [GTMAENIPSSA], 2019), no final do ano de 2019 existiam cerca de 7107 pessoas em situação de sem-abrigo no território nacional (GTMA-ENIPSSA, 2019). Perante a realidade destes dados importa sublinhar a importância da sensibilização para este fenómeno.

A disseminação e consciência do conceito de pessoa em situação de sem-abrigo é de extrema importância. Um dos objetivos centrais da Estratégia Nacional para a Integração das Pessoas em Situação de Sem-Abrigo (GTMAENIPSSA, 2019) é tornar universal este conceito (Resolução do Conselho de Ministros, n. 107/2017). Por vezes, coloquialmente, é usado apenas o termo sem-abrigo - no entanto, é importante compreender que a pessoa não é sem-abrigo, está sem-abrigo. Corresponde a uma condição, uma circunstância, e não a uma característica intrínseca que se possa traduzir numa definição pessoal.

Além disso, importa referir a multidimensionalidade deste fenómeno. De acordo com Somerville (2013), não se trata apenas da falta de abrigo - uma morada, um teto - e sim da privação de vários elementos como: bem-estar fisiológico (falta de conforto corporal ou calor), emocional (falta de amor ou alegria), territorial (falta de privacidade), ontológica (falta de pertença no mundo) e espiritual (falta de esperança, falta de propósito). Desta forma, ter consciência da amplitude do fenómeno é essencial para a promoção de estratégias e medidas que vão ao encontro das (reais) necessidades destas pessoas. Ainda, numa perspetiva social, sensibilizar e educar a população geral para a abrangência do fenómeno torna-se pertinente numa 
tentativa de minimização da exclusão social. Segundo Aldeia (2014, p. 22), as pessoas em situação de semabrigo não correspondem à visão de "seres estranhos, fundamentalmente diferentes da normalidadepadrão", considerando que são essas narrativas, negativas e repressivas, que perpetuam o status quo do fenómeno.

O conceito de atitude, embora amplamente disseminado e investigado, não possui uma definição consensual. Após uma revisão de literatura destacamos três definições:

"Atitude é um estado de preparação mental ou neural, organizado através da experiência, e exercendo uma influência dinâmica sobre as respostas individuais a todos os objetos ou situações com que se relaciona" (Allport, 1935, citado por Lima \& Correia, 2013, p. 203).

"Atitude é uma ideia carregada de emoção que predispõe um conjunto de ações a um conjunto particular de situações sociais" (Triandis, 1971, citado por Neiva \& Mauro, 2011, p. 174).

“Atitude é um construto hipotético referente à tendência psicológica que se expressa numa avaliação favorável ou desfavorável de uma entidade específica” (Eagly \& Chaiken, 1993, p. 1).

As definições supracitadas fornecem diretrizes importantes acerca do fenómeno das atitudes. Na primeira definição é indicado que as atitudes se organizam através da experiência, ou seja, as experiências prévias individuais influenciam e contribuem para a formação de atitudes, tendo um papel relevante. A segunda definição inclui o modelo tripartido das atitudes (componente cognitiva, emocional e comportamental) numa visão dinâmica - as componentes interagem entre si. A terceira definição, comummente aceite na literatura, indica as atitudes como um construto hipotético que, segundo Lima e Correia (2013), traduz a ideia de que as atitudes não são diretamente observáveis, mas inferidas de respostas observáveis (Neiva \& Mauro, 2011), ou seja, inferidas através de respostas comportamentais. Além disso, a última afirmação dá-nos uma ideia de avaliação e de posterior posicionamento, isto é, a atitude acaba por ser uma posição resultante da avaliação que o indivíduo faz das circunstâncias. Nesta linha de pensamento, Lima e Correia (2013) consideram que um dos pontos de harmonia entre as diversas definições existentes reside no facto de as atitudes se expressarem sempre através de um julgamento avaliativo. Sendo o espectro atitudinal um continuum, é extremamente difícil a existência de uma posição neutra relativamente a um assunto. Assim, a única situação em que tal é possível, segundo Neiva e Mauro (2011), ocorre apenas quando o indivíduo desconhece a existência do fenómeno (não sabe o que é, nunca viu, nem ouviu falar). Neste sentido, qualquer conhecimento, independentemente da sua intensidade ou força, posiciona-o no continuum atitudinal, adotando um posicionamento favorável ou desfavorável (direção de atitude). De forma mais clara, de acordo com Lima e Correia (2013), as características inerentes ao julgamento avaliativo (atitude), são: a) a direção (favorável ou desfavorável); b) a intensidade (posições extremas/radicais e posições mais fracas, por comparação); e c) a acessibilidade (probabilidade de ser atividade automaticamente), que está diretamente relacionada com a força com que foi aprendida e com a frequência com que é usada. Neste sentido e a título de exemplo, duas pessoas podem apresentar uma atitude na mesma direção favorável, mas com intensidades distintas, ou seja, podem concordar que uma pessoa em situação de sem-abrigo pode dormir em locais públicos, mas uma delas considera que só o deve fazer durante a noite.

No que respeita aos componentes das atitudes, Lima e Correia (2013) indicam que a base teórica proeminente na literatura assenta no modelo de três componentes ou modelo tripartido, integrando as componentes 
cognitiva, afetiva e comportamental, que apesar de teoricamente distintas, são interdependentes, influenciando-se e reforçando-se mutuamente. De referir também que estes mesmos autores consideram que não é necessário que uma atitude seja representada pelas três componentes (pode existir uma atitude unicamente afetiva, por exemplo). Na Figura 1 é possível observar um exemplo ilustrativo do modelo tripartido.

\section{Figura 1}

Componentes do Modelo Tripartido de Atitudes

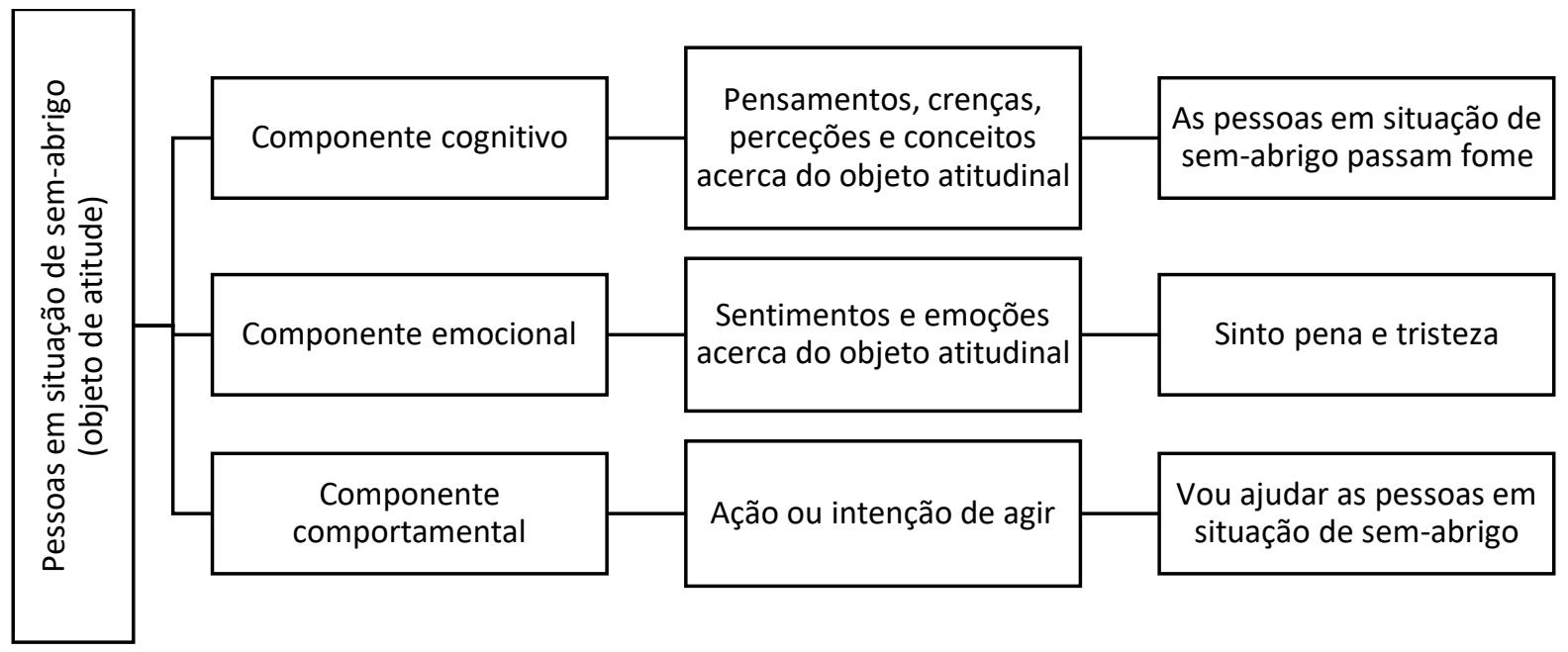

Outro ponto que merece destaque é a distinção entre atitudes implícitas e explícitas. Bizarrias e Brandão (2017) sugerem que perante um mesmo objeto atitudinal, as atitudes podem ser duplas, isto é, espontâneas ou deliberadas. Tal significa que as atitudes implícitas derivam de um processo espontâneo ou automático, enquanto as atitudes explícitas resultam de um processo consciente. Na mesma linha de pensamento, Greenwald et al. (2002) referem que as atitudes explícitas representam o que o indivíduo está disposto ou é capaz de declarar, ao contrário das implícitas que estão fora do controlo e podem revelar preferências que o próprio desconhece ou não quer declarar (Greenwald \& Banaji, 1995; Greenwald et al., 2002). Greenwald e Banaji (1995) hipotetizam que as atitudes implícitas derivam de experiências passadas, das quais as pessoas têm pouca ou nenhuma consciência, mas que influenciam os processos cognitivos, afetivos e comportamentais em relação ao objeto de atitude. Neste sentido, de forma a medir as atitudes implícitas, Greenwald et al. (1998) desenvolveram o Teste de Associação Implícita (TAI), que avalia a latência de resposta na associação de estímulos a uma categoria, possibilitando respostas que estejam fora da intencionalidade do participante, minimizando o efeito da desejabilidade social (Gouveia et al., 2014). De notar que o TAl é apenas uma das formas de avaliar as atitudes implícitas, existindo outras, como as técnicas projetivas, o priming afetivo, medidas indiretas de observação comportamental, medidas de natureza corporal, entre outras (Lima \& Correia, 2013).

Apesar de existirem instrumentos que medem atitudes em relação às pessoas em situação de sem-abrigo, muitos derivam da tradução e/ou adaptação do instrumento desenvolvido por Toro e McDonell (1992) — 
Beliefs, Attitudes, and Knowledge About Homelessness - pioneiro neste campo. Importa referir que este instrumento e as suas adaptações (Miguel et al., 2010; Petit et al., 2018; Tompsett et al., 2006; Toro et al., 2007; Tsai et al., 2018), além de explorarem outros constructos que não somente atitudes (e.g., conhecimentos, crenças, opiniões, causas percebidas para o fenómeno, soluções, etc.) apresentam uma extensão considerável, o que torna a investigação extensa e morosa.

De referir ainda que outras medidas, desenvolvidas para a medição de atitudes face a estas pessoas são destinadas a populações especificas, como profissionais de saúde ou estudantes (Copeland et al., 2020; Klarare et al., 2020; Lester \& Pattison, 2000; Phillips, 2015; Wisehart et al., 2013), nem sempre evidenciam bons índices psicométricos ou, também, não se focam somente na medição de atitudes (Agans \& Liu, 2015; Cruz, 2018; Guzewicz \& Takooshian, 1992; Hocking \& Lawrence, 2000; Krajewska-Kułak et al., 2016), não disponibilizando, assim, uma medida breve. Importa realçar, igualmente, o facto de a maioria das escalas ou medidas de atitudes nesta população se focalizarem, unicamente, na dimensão cognitiva das atitudes (Snow-Hill, 2019), o que contraria a sugestão de Fiske (2018), que indica que a distinção das dimensões atitudinais potencia uma maior compreensão da complexidade das atitudes. Na mesma linha de pensamento, Snow-Hill (2019) considera que seria útil o desenvolvimento de um modelo que identificasse especificamente a componente cognitiva, comportamental e emocional, de forma a criar uma medição mais adequada das atitudes em relação às pessoas em situação de sem-abrigo.

Importa destacar que as três componentes atitudinais assemelham-se às três dimensões do modelo cognitivocomportamental (Wright et al., 2019). Na Figura 2 é possível verificar que o mesmo diagrama serve, simultaneamente, para representar o modelo cognitivo-comportamental (Wright et al., 2019) e o modelo tripartido das atitudes. Esta semelhança associa-se a uma das pretensões deste estudo, ou seja, ao compreender a relação entre as componentes atitudinais, também se pode tornar mais fácil a compreensão e intervenção dirigida a promover mudanças cognitivas, emocionais ou comportamentais.

\section{Figura 2}

Semelhança Teórica entre o Modelo tripartido de Atitudes e o Modelo Cognitivo-Comportamental

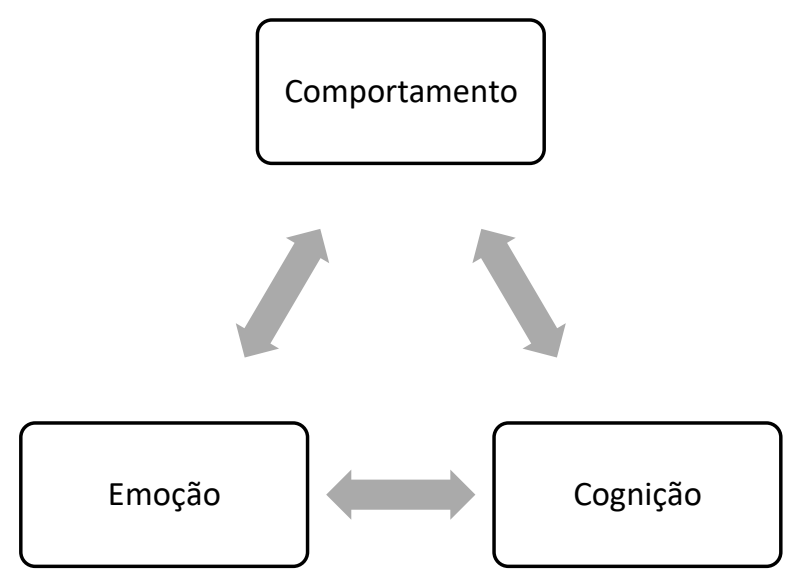


Relativamente a estudos anteriores que se tenham debruçado sobre atitudes dirigidas especificamente à população em situação de sem-abrigo, Petit et al. (2019), ao compararem as atitudes e opiniões da população geral de vários países acerca do fenómeno, referem que as pessoas consideraram que a despesa pública e os programas de combate a este fenómeno foram insuficientes. Porém, os entrevistados demonstraram relutância em pagar mais impostos para reduzir a incidência de casos de pessoas em situação de sem-abrigo. Ainda, uma proporção considerável dos participantes considerou que a população em situação de sem-abrigo permanece na rua por opção - taxas mais elevadas na Polónia (79,9\%) e em Portugal (67,6\%). Por sua vez, o estudo conduzido por Tsai et al. (2018) demonstrou que a maioria dos participantes afirmava sentir compaixão em relação às pessoas em situação de sem-abrigo, concordando que estas pudessem dormir em locais públicos (7093\%), ainda que um menor número de participantes (49-59\%) concordasse com a possibilidade de acamparem na rua ou de pedirem esmolas em espaços públicos. De acrescentar que neste estudo foram também relatados medos e preocupações sobre a perigosidade das pessoas em situação de sem-abrigo (Tsai et al., 2018). Esta eventual associação com a criminalidade foi igualmente reportada num outro estudo, realizado na população estudantil polaca (Krajewska-Kułak et al., 2016).

No que respeita às atitudes em relação às pessoas em situação de sem-abrigo em Portugal, existem poucos estudos que contemplem as atitudes e as suas componentes. Um estudo realizado por Miguel et al. (2010) concluiu que a maioria dos participantes considerou que as pessoas em situação de sem-abrigo têm boas capacidades de trabalho e que não confiaria menos numa pessoa se soubessem que ela tinha estado sem-abrigo. No entanto, os participantes deste mesmo estudo consideraram também que as pessoas sem-abrigo têm mais probabilidades de cometer crimes violentos (Miguel et al., 2010). Um estudo mais recente (Cruz, 2018) investigou a opinião de pessoas sem-abrigo acerca da perspetiva que consideram que é adotada pela sociedade perante o fenómeno dos sem-abrigo. Relativamente a esta dimensão, os resultados a pontaram para a presença de estereótipos e discriminação social em relação à população em situação de sem-abrigo.

Neste contexto, o presente estudo pretendeu desenvolver uma primeira versão de um questionário breve, focado nas atitudes e contemplando as dimensões comportamental, emocional e cognitiva. A existência de um instrumento válido e fidedigno desenhado para este efeito possibilitará o desenvolvimento de mais investigação que, por sua vez, poderá facultar informações relevantes para a criação de medidas ajustadas para a promoção de atitudes mais favoráveis relativamente à população em situação de sem-abrigo.

\section{Método}

\section{Participantes}

Participaram no presente estudo 361 sujeitos com idade superior ou igual a 18 anos. Como é possível verificar na Tabela 1, a amostra integrou 204 (56,5\%) pessoas do sexo feminino e 157 (43,5\%) do sexo masculino. A média de idades foi de 26,98 ( $D P=7,42)$ anos. 


\section{Tabela 1}

Caracterização Sociodemográfica da Amostra

\begin{tabular}{lcc}
\hline & $n$ & $\%$ \\
\hline Sexo & 204 & 56,5 \\
Feminino & 157 & 43,5 \\
Masculino & & 87,5 \\
Estado civil & 316 & 10,8 \\
Solteiro & 39 & 1,7 \\
Casado/a ou União de facto & 6 & - \\
Divorciado/a ou Separado/a & - & \\
Viúvo/a & & 186 \\
Situação Profissional & 19 & 51,5 \\
Empregado a full-time & 38 & 5,3 \\
Empregado a part-time & 102 & 10,5 \\
Desempregado & - & 28,3 \\
Estudante & 16 & - \\
Reformado & & 4,4 \\
Outra & &
\end{tabular}

Nota. $N=361$.

Relativamente ao estado civil, a maioria dos participantes era solteiro $(n=316 ; 87,5 \%)$. A média dos anos de escolaridade foi de 13,76 ( $D P=2,47)$, apresentando um mínimo de três e um máximo de 23 anos. Em relação à situação profissional, 186 (51,5\%) participantes indicaram que estavam empregados a full-time, 102 $(28,3 \%)$ eram estudantes e $38(10,5 \%)$ encontravam-se desempregados.

\section{Instrumentos}

No presente estudo foram usados os instrumentos de autorresposta que a seguir se descrevem.

\section{Questionário sociodemográfico}

Foi criado um breve questionário com as variáveis idade, sexo, estado civil, escolaridade e situação profissional.

\section{Questionário de Atitudes em Relação à População em Situação de Sem-Abrigo (QARPSS)}

Este instrumento foi desenvolvido como objetivo nuclear do presente trabalho, pretendendo avaliar as diferentes componentes das atitudes em relação à população em situação de sem-abrigo. Desta forma, é constituído por três dimensões - comportamental, cognitiva e emocional - que representam o modelo tripartido das atitudes. As dimensões cognitiva (e.g., Penso que a maioria das pessoas em situação de sem-abrigo... está na rua por vontade própria) e emocional (e.g., Na presença de uma pessoa em situação de sem-abrigo sinto... medo de ser contagiado com doenças) integram 11 itens cada e a dimensão comportamental é composta por dez itens (e.g., Quando vejo uma pessoa em situação de sem-abrigo... sorrio para ele/a). Para cada item é pedido aos respondentes que indiquem o seu grau de concordância com as afirmações, numa escala de cinco pontos, indo de discordo totalmente (1) a concordo totalmente 
(5). O questionário contém afirmações em polos distintos (positivo e negativo), o que proporciona uma análise do continuum do espectro atitudinal. Pontuações mais elevadas representam atitudes mais positivas.

\section{Inventário de Atitudes face à Procura de Serviços de Saúde Mental (IATSMHS)}

O IATSMHS (Inventory of Attitudes toward Seeking Mental Health Services; Mckenzie et al., 2004; versão portuguesa de Fonseca et al., 2017) é um instrumento de autorresposta com 24 itens, desenvolvido para avaliar as atitudes em relação à procura de serviços de saúde mental. É composto por três dimensões: Abertura Psicológica (e.g., As pessoas devem resolver os seus próprios problemas; receber ajuda profissional deve ser o último recurso), Propensão para a Procura de Ajuda (e.g., Se eu tivesse um problema psicológico grave nesta fase da minha vida, iria achar que a psicoterapia me ajudaria) e Indiferença ao Estigma (e.g., Ter sido diagnosticado com uma perturbação mental é uma nódoa na vida de uma pessoa). De acordo com as autoras da versão portuguesa, a escala de resposta varia ao longo de cinco possibilidades, de discordo (0) a concordo (4). No que respeita à interpretação dos resultados, pontuações mais elevadas correspondem a atitudes mais positivas (Fonseca et al., 2017). Em relação aos indicadores psicométricos, na versão original o coeficiente de alfa de Cronbach para a escala total foi de 0,87 (Mackenzie et al., 2004) e na versão portuguesa de 0,83 (Fonseca et al., 2017). No presente estudo o alfa de Cronbach foi de 0,72.

\section{Escalas de Motivação Interna (EMI) e Motivação Externa (EME) para Responder sem Preconceito}

As EMI e EME (Internal and External Motivation to Respond Without Prejudice; Plant \& Devine, 1998; versão portuguesa de Palma \& Maroco, 2008) foram desenvolvidas considerando que ambos os tipos de motivação — interna e externa - afetam as reações preconceituosas e não-preconceituosas das pessoas (Palma \& Maroco, 2008). Por sua vez, a versão portuguesa é composta por 8 itens - 4 itens na EMI (e.g., Devido aos meus valores pessoais creio que é errado usar estereótipos acerca das pessoas negras) - e 4 itens na EME (e.g., Tento agir de forma não-preconceituosa face às pessoas negras devido à pressão de outras pessoas). De acordo com Palma e Maroco (2008), é pedido aos participantes que indiquem o grau de concordância numa escala que varia entre discordo fortemente (1) e concordo fortemente (9). Pontuações mais elevadas indicam maior motivação interna ou externa. Importa destacar que estas subescalas são direcionadas para o preconceito relativo ao racismo pelo que foi necessário realizar uma adaptação frásica, relacionando os itens com a população em situação de sem-abrigo (e.g., Devido aos meus valores pessoais creio que é errado usar estereótipos acerca das pessoas em situação de sem-abrigo), de forma a incluir este instrumento no presente estudo. A versão original deste instrumento apresentou valores de consistência interna a variar entre 0,76 e 0,85 para as duas subescalas (Plant \& Devine, 1998). Por sua vez, a versão portuguesa obteve valores de alfa de Cronbach de 0,67 (EMI) e de 0,83 (EME) (Palma \& Maroco, 2008). No presente estudo observaram-se valores de alfa de Cronbach de 0,56 na Escala de Motivação Interna e de 0,81 na Escala de Motivação Externa.

\section{Escala de Distância Social (EDS)}

A EDS (Social Distance Scale; Bogardus, 1933) original traduz-se numa escala cumulativa, onde perante um conjunto típico de afirmações numeradas, com níveis de proximidade graduais, o participante deve selecionar se aceitaria aquela afirmação (e.g., Aceitar uma pessoa em situação de sem-abrigo como vizinho ou Aceitar uma pessoa em situação de sem-abrigo como companheiro). Esta versão parte de pressuposto que o respondente, ao aceitar um nível mais alto de intimidade, também aceita os anteriores (Wark \& Galliher, 2007). Desta forma, o seu nível de distância social perante o grupo é medido pelo nível em que deixou de aceitar alguma afirmação. No entanto, atualmente, são usadas outras formas de medir o construto de Distância Social. Um estudo realizado por Mather et al. (2017) demonstrou que incluir uma medida de intensidade no instrumento 
apresenta resultados mais sensíveis e representativos da realidade. Os autores usaram a escala tradicional de Bogardus (1933) e adicionaram uma escala de Likert de cinco pontos. O nível de desejabilidade social é calculado multiplicando os níveis das questões tradicionais pela resposta dada na escala de Likert, podendo variar entre 28 (níveis x 1) e 140 pontos (níveis x 5). Apesar de não ter sido calculado o nível de consistência interna, os resultados deste estudo indicam que a forma mais adequada de cotação reside na complementaridade entre os níveis tradicionais e o método iScore (intensidade). Ainda, Mather et al. (2017) salientam que o método tradicional se torna redutor por não englobar os casos de participantes ambivalentes, forçando-os a responder de forma dicotómica. Os mesmos autores, levantam outra questão, sugerindo que a ordem de proximidade gradual se torna um fator de enviesamento porque representa a perspetiva de proximidade do investigador 0 que não corresponde, por vezes, à visão do participante (Mather et al., 2017). A EDS revelou, no presente estudo, um valor de alfa de Cronbach de 0,86 .

\section{Escala de Desejabilidade Social de 20 itens (EDS-20)}

A EDS-20 (Almiro et al., 2017), tal como o nome indica, é constituída por 20 itens que avaliam a desejabilidade social, os quais são respondidos dicotomicamente com sim/não. No que respeita à cotação, a maioria dos itens é de sentido inverso pelo que a resposta sim é cotada com zero pontos e a resposta não é cotada com um ponto. A pontuação total indica o nível de desejabilidade social do respondente, que pode variar entre zero e 20 pontos e, pontuações mais elevadas indicam um maior nível de desejabilidade social. Em termos psicométricos, a EDS-20 apresenta bons índices de consistência interna ( $\alpha$ de Cronbach = 0,85), tratando-se de uma medida unidimensional (Almiro et al., 2017). No presente estudo o alfa de Cronbach foi indicador de uma boa consistência interna $(\alpha=0,78)$.

\section{Procedimentos Metodológicos}

Atendendo aos objetivos do estudo, foram selecionados outros instrumentos de autorresposta e solicitada a autorização para a sua utilização aos autores das respetivas versões portuguesas. Uma vez obtida a referida autorização foi composto um protocolo de avaliação que integrou os instrumentos acima elencados e que foi colocado na plataforma Google Forms. O recrutamento dos participantes foi efetuado através da divulgação do estudo nas redes sociais Facebook, Instagram e WhatsApp, correspondendo a uma amostragem não probabilística por bola de neve. Como critério de inclusão foi definida a idade igual ou superior a 18 anos. Na divulgação do estudo, a parte inicial contemplou um texto informativo acerca do âmbito e objetivos do mesmo, a garantia de confidencialidade e anonimato e a possibilidade de os participantes desistirem a qualquer momento. Os participantes apenas acediam ao protocolo de avaliação após assinalarem três declarações relativas aos critérios de inclusão e ao consentimento informado ("Declaro que participo, de forma voluntária, nesta investigação."; "Declaro que tenho mais de 18 anos"; "Declaro que autorizo o uso dos dados fornecidos para fins de investigação"). A recolha de dados realizou-se entre novembro de 2020 e março de 2021.

\section{Desenvolvimento do Questionário de Atitudes em Relação à População em Situação de Sem-Abrigo}

Após uma revisão de literatura acerca dos estudos existentes sobre as atitudes em relação à população em situação de sem-abrigo, desenvolveu-se o QARPSSA. Este teve como base teórica o Modelo Tripartido das Atitudes, tendo sido, inicialmente, elaborada uma pool constituída por 36 itens. Porém, após discussão realizada com um painel de três psicólogos com experiência na construção de instrumentos de avaliação e domínio dos constructos envolvidos no QARPSSA, foi observado que alguns dos itens, poderiam ser interpretados como correspondendo, por exemplo, a 
cognições ou a emoções. Nesta sequência, foram eliminados quatro itens e alterada a construção frásica de dois. De modo a evitar que o texto de cada item fosse demasiado extenso e que a construção frásica dos diferentes itens apresentasse repetições (e.g., "Quando vejo"; "Penso que..."), optou-se por dividir os itens em três dimensões teóricas com uma expressão inicial, que se adaptasse a todos os itens dessa dimensão. Assim, para a dimensão comportamental iniciaramse os itens com "Quando vejo uma pessoa em situação de sem-abrigo...", na dimensão emocional com "Quando vejo uma pessoa em situação de sem-abrigo sinto..." e para a dimensão cognitiva "Penso que a maioria das pessoas em situação de sem-abrigo...". Com vista à exploração da compreensibilidade dos itens, foi solicitado a um conjunto de dez pessoas (cinco estudantes e cinco pessoas da população geral, das relações dos investigadores) que preenchessem o QARPSSA e se pronunciassem relativamente à clareza das instruções e dos itens, não tendo sido reportadas quaisquer dificuldades, pelo que o seu conteúdo e redação foram mantidos.

\section{Procedimentos Analíticos}

Para a análise descritiva e psicométrica dos dados, foi utilizado o software Statistical Package for the Social Sciences (IBM SPSS Statistics, versão 26) e para a análise fatorial confirmatória (AFC) o software IBM SPSS AMOS (versão 25). Realizou-se uma análise descritiva das variáveis sociodemográficas sendo calculadas medidas de tendência central e de dispersão para as variáveis contínuas e frequências e percentagens para as variáveis categoriais. Com o objetivo de realizar uma análise de componentes principais $(A C P)$, recorreu-se ao uso do teste $K M O$ e do teste de esfericidade de Bartlett, de forma a avaliar a adequação dos dados. $O$ tamanho da amostra $(N=361)$ revelou-se adequado para a condução desta análise (DeVellis, 2011). Foram retidos os itens que revelaram pesos fatoriais superiores a 0,40 . $\mathrm{Na}$ análise fatorial confirmatória (AFC), foram tidos em conta os seguintes índices de ajustamento: o qui-quadrado normalizado ( $\left.\chi^{2} / g l\right)$; o Comparative Fit Index (CFI); o Goodness of Fit Index (GFI); o Root Mean Square Error of Approximation o (RMSEA); e para comparação dos modelos, o Minor Expected Cross-Validation Index (MECVI). De acordo com Marôco (2010), valores de CFI e de GFI de 0,90 a 0,95 e valores de 0,05 a 0,08 do RMSEA são indicadores de um bom ajustamento. Relativamente ao MECVI, foi considerado como revelando um melhor ajustamento o modelo com o valor mais baixo (Marôco, 2010). Para a análise da presença de outliers foram observadas as distâncias de Mahalanobis ao quadrado $\left(D M^{2}\right)$. Os índices de ajustamento local dos itens foram avaliados e considerados adequados sempre que o seu valor se mostrou superior a 0,40 (Tabachnick \& Fidell, 2007). No que respeita à avaliação da fidedignidade dos itens do QARPSSA, foi calculado o alfa de Cronbach e as correlações item-total. Valores de alfa de Cronbach superiores a 0,70 correspondem a uma boa fidedignidade e valores superiores a 0,90 são reveladores de uma fidedignidade excelente (Kline, 2000). Adicionalmente, recorreu-se ao cálculo da fiabilidade composta (FC) e da variância extraída média (VEM) de modo a confirmar a validade de construto e convergente. Para cálculo da $F C$ recorreu-se a uma calculadora da fiabilidade compósita (estima a $F C$ com base nos pesos fatoriais estandardizados e nas variâncias de erro; Raykov, 1997; https://bit.ly/3fuMocV). A VEM foi calculada manualmente através da respetiva fórmula de cálculo (Fornell \& Larcker, 1981). Para efeitos de análise da fidedignidade teste-reteste, procedeu-se ao cálculo de testes $t$ para amostras emparelhadas e do coeficiente de correlação de Pearson numa subamostra $(n=39)$, sendo considerado um intervalo de oito semanas. De forma a explorar as relações entre o QARPSSA e as demais variáveis recorreu-se aos coeficientes de correlação de Pearson. Os valores de referência para as correlações foram os indicados por Cohen et al. (2003), que considera que as correlações podem ser consideradas pequenas $(r=0,10$ a 0,29$)$, moderadas ( $r=0,30$ a 0,49), grandes $(r=0,50$ a 0,69$)$, muito grandes $(r=0,70$ a 0,89), quase perfeitas $(r \geq 0,90)$ e perfeitas $(r=1)$. Por último, foram calculados teste $t$ para amostras independentes com o intuito de explorar a eventual existência de diferenças entre homens e mulheres da presente amostra no que se refere ao score total do QARPSSA. 


\section{Resultados}

Relativamente ao questionário central neste estudo, o QARPSSA, foram analisadas as médias, desvios-padrão, correlações item-total e alfa de Cronbach se item removido da pool inicial de 32 itens (Tabela 2).

\section{Tabela 2}

Estatísticas de Confiabilidade dos Itens da Pool Inicial do QARPSSA (32 itens)

\begin{tabular}{|c|c|c|c|}
\hline & $M(D P)$ & $r$ Item-total & $\begin{array}{l}\alpha \text { de Cronbach } \\
\text { se item removido }\end{array}$ \\
\hline \multicolumn{4}{|l|}{ Dimensão Comportamental } \\
\hline 1. Desvio o olhar e finjo que não o/a vi & $1,91(1,00)$ & 0,33 & 0,82 \\
\hline 2. Prefiro não olhar porque me custa & $2,61(1,30)$ & 0,35 & 0,82 \\
\hline 3. Passo/ prefiro passar para o outro lado da rua & $1,48(0,85)$ & 0,36 & 0,82 \\
\hline 4. Escondo objetos de valor & $1,55(0,90)$ & 0,41 & 0,82 \\
\hline 5. Sorrio para ele/a & $2,96(1,12)$ & 0,06 & 0,83 \\
\hline 6. Procuro ajudar (dou comida, dinheiro, roupa ou outros bens) & $3,27(1,00)$ & 0,06 & 0,83 \\
\hline 7. Tento conversar com ele/a, numa tentativa de ajudar & $2,54(1,10)$ & 0,10 & 0,83 \\
\hline 8. Não sei o que fazer e acabo por não fazer nada & $2,91(1,28)$ & 0,32 & 0,82 \\
\hline 9. Vejo se estão pessoas por perto (polícia, ou pessoas que possam ajudar) & $2,22(1,14)$ & 0,29 & 0,82 \\
\hline $\begin{array}{l}\text { 10. Fico a olhar/observar a pessoa para perceber se é seguro aproximar-me } \\
\text { ou não }\end{array}$ & $2,16(1,14)$ & 0,40 & 0,82 \\
\hline \multicolumn{4}{|l|}{ Dimensão Emocional } \\
\hline 11. Receio que me peça dinheiro & $2,36(1,29)$ & 0,51 & 0,81 \\
\hline 12. Medo de ser assaltado/a & $1,93(1,13)$ & 0,49 & 0,81 \\
\hline 13. Que sou superior a ele/a & $1,16(0,55)$ & 0,28 & 0,82 \\
\hline 14. Nervosismo ou ansiedade & $1,87(1,10)$ & 0,43 & 0,81 \\
\hline 15. Pena dele/a & $3,64(1,13)$ & 0,36 & 0,82 \\
\hline 16. Sensibilidade ao seu sofrimento & $4,00(0,97)$ & 0,16 & 0,82 \\
\hline 17. Tristeza por não o/a poder ajudar & $3,77(1,08)$ & 0,19 & 0,82 \\
\hline 18. Indiferença e continuo com a minha vida & $1,64(0,91)$ & 0,24 & 0,82 \\
\hline 19. Nojo/repulsa & $1,20(0,56)$ & 0,41 & 0,82 \\
\hline 20. Medo de ser contagiado com doenças & $1,32(0,74)$ & 0,43 & 0,82 \\
\hline 21. Admiração & $2,27(1,17)$ & 0,06 & 0,83 \\
\hline \multicolumn{4}{|l|}{ Dimensão Cognitiva } \\
\hline 22. Está na rua por vontade própria & $1,97(1,04)$ & 0,32 & 0,82 \\
\hline 23. Não tem capacidades para manter um trabalho fixo & $2,43(1,19)$ & 0,32 & 0,82 \\
\hline $\begin{array}{l}\text { 24. Tem mais probabilidades de cometer crimes (violentos) comparativamente } \\
\text { com as outras pessoas }\end{array}$ & $2,02(1,04)$ & 0,42 & 0,81 \\
\hline 25. Sente dificuldades em fazer amigos ou manter relacionamentos sociais & $3,12(1,28)$ & 0,33 & 0,82 \\
\hline 26. É vítima de circunstâncias sociais adversas & $3,81(1,04)$ & 0,13 & 0,82 \\
\hline 27. Não procura mudar de vida & $2,51(1,00)$ & 0,45 & 0,81 \\
\hline 28. Tem problemas com álcool/drogas & $3,04(1,02)$ & 0,52 & 0,81 \\
\hline 29. Pede na rua ou rouba e, apenas por isso, é que tem dinheiro & $2,20(1,02)$ & 0,45 & 0,81 \\
\hline 30. Apresenta algum tipo de perturbação mental & $2,47(1,07)$ & 0,39 & 0,82 \\
\hline $\begin{array}{l}\text { 31. Habitua-se a estar na rua e acaba por não procurar outras alternativas } \\
\text { para a sua vida }\end{array}$ & $2,84(1,15)$ & 0,52 & 0,81 \\
\hline 32. São pessoas em quem não se pode confiar & $1,84(0,91)$ & 0,46 & 0,81 \\
\hline
\end{tabular}

Nota. $N=$ 361. QARPSSA = Questionário de Atitudes em Relação à População em Situação de Sem-Abrigo. 
Com o objetivo de testar a estrutura fatorial do QARPSSA, recorreu-se à verificação dos valores do teste de $K M O$, e do teste de esfericidade de Bartlett. O KMO foi de 0,83 e o teste de esfericidade de Bartlett mostrouse significativo $\left[\chi^{2}{ }_{(496)}=4059 ; p<0,001\right]$, indicando a adequação da ACP. Esta análise, forçada a três fatores, de acordo com o modelo teórico que serviu de base à elaboração da pool de itens, conduziu à eliminação de 15 itens, da pool inicial de 32 itens, que revelaram pesos fatoriais inferiores a 0,40 e/ou que saturaram em mais que um fator, ficando o QARPSSA composto por 17 itens (Tabela 3). Todos os itens se agruparam na dimensão expetável de acordo com o modelo teórico, à exceção do item 21 (Quando vejo uma pessoa em situação de sem-abrigo sinto admiração) que foi considerado, originalmente, como pertencente à dimensão emocional. Os valores de correlação item-total oscilaram entre 0,35 (Item 22) e 0,52 (Item 28) na dimensão cognitiva, entre 0,39 (Item 11) e 0,46 (Itens 12 e 20) na dimensão emocional e entre 0,10 (Itens 5, 6 e 21) e 0,33 (Item 9) na dimensão comportamental. Ainda que os Itens 5, 6 e 21 apresentassem valores de correlação com o total pequenos, optou-se por mantê-los em virtude da sua relevância teórica.

Com o intuito de avaliar o ajustamento dos dados da versão de 17 itens do QARPSSA a um modelo de ordem superior composto por três fatores relativos às cognições, emoções e comportamentos (modelo teórico), procedeu-se a uma análise fatorial confirmatória $(A F C)$. O modelo testado revelou os seguintes valores no que respeita aos indicadores de ajustamento: qui-quadrado normalizado $\chi^{2} / g I=2,94 ; C F I=0,87 ; G F I=0,90$; $R M S E A=0,07$ e $M E C V I=1,16$. Analisadas as distâncias de Mahalanobis ao quadrado $\left(D M^{2}\right)$, observou-se a presença de várias observações correspondentes a outliers, contudo, optou-se por não as eliminar atendendo a que a variabilidade associada aos fatores em estudo reduziria possíveis interpretações de utilidade nesta análise. Relativamente à normalidade da distribuição dos itens, nenhum deles evidenciou valores de $S k$ e de $K u$ reveladores de violações severas à distribuição normal $(|S k|<3$ e $|K u|<10)$. A análise dos índices de modificação indicou que os resíduos dos Itens 19 e 20 se encontravam correlacionados. Nesta sequência foi definida a correlação entre estes resíduos e o modelo foi recalculado. Este modelo evidenciou uma boa qualidade de ajustamento: $C F I=0,90 ; G F I=0,92 ; R M S E A=0,07$ e $M E C V I=1,02$. O valor do MECVI, usado para comparação dos modelos, foi menor no modelo recalculado. Apenas o índice do ajustamento do quiquadrado normalizado apresentou um valor sofrível $\left(\chi^{2} / g l=2,51\right)$. Os indicadores de ajustamento local encontram-se apresentados na Tabela 3.

Como se pode verificar, os pesos fatoriais oscilaram entre 0,38 (Item 21) e 0,83 (Item 7) e as correlações múltiplas ao quadrado indicaram valores entre 0,14 (Item 21) e 0,69 (Item 7). A VEM foi de 0,49, valor superior ao quadrado das correlações dos fatores que variou entre 0,002 e 0,25 , sendo sugestivo de validade discriminante. Os 17 itens que constituem os três fatores - comportamental, emocional e cognitivo explicaram $50,7 \%$ da variância. 


\section{Tabela 3}

Pesos Fatoriais e Correlações Múltiplas ao Quadrado dos Itens do QARPSSA (17 itens) Resultantes da AFC

\begin{tabular}{|c|c|c|}
\hline & Pesos fatoriais & $r^{2}$ \\
\hline \multicolumn{3}{|l|}{ Dimensão Comportamental } \\
\hline \multicolumn{3}{|l|}{ Quando vejo uma pessoa em situação de sem-abrigo... } \\
\hline 5. Sorrio para ele/a & 0,60 & 0,36 \\
\hline 6. Procuro ajudar (dou comida, dinheiro, roupa ou outros bens) & 0,63 & 0,40 \\
\hline 7. Tento conversar com ele/a, numa tentativa de ajudar & 0,83 & 0,69 \\
\hline 9. Vejo se estão pessoas por perto (polícia, ou pessoas que possam ajudar) & 0,44 & 0,19 \\
\hline 21. Admiração & 0,38 & 0,14 \\
\hline \multicolumn{3}{|l|}{ Dimensão Emocional } \\
\hline \multicolumn{3}{|l|}{ Na presença de uma pessoa em situação de sem-abrigo sinto... } \\
\hline 11. Receio que me peça dinheiro & 0,66 & 0,44 \\
\hline 12. Medo de ser assaltado/a & 0,74 & 0,55 \\
\hline 19. Nojo/repulsa & 0,49 & 0,24 \\
\hline 20. Medo de ser contagiado com doenças & 0,55 & 0,31 \\
\hline \multicolumn{3}{|l|}{ Dimensão Cognitiva } \\
\hline \multicolumn{3}{|l|}{ Penso que a maioria das pessoas em situação de sem-abrigo... } \\
\hline 22. Está na rua por vontade própria & 0,51 & 0,27 \\
\hline $\begin{array}{l}\text { 24. Tem mais probabilidades de cometer crimes (violentos) comparativamente } \\
\text { com as outras pessoas }\end{array}$ & 0,54 & 0,30 \\
\hline 27. Não procura mudar de vida & 0,71 & 0,50 \\
\hline 28. Tem problemas com álcool/drogas & 0,69 & 0,48 \\
\hline 29. Pede na rua ou rouba e, apenas por isso, é que tem dinheiro & 0,68 & 0,46 \\
\hline 30. Apresenta algum tipo de perturbação mental & 0,54 & 0,30 \\
\hline $\begin{array}{l}\text { 31. Habitua-se a estar na rua e acaba por não procurar outras alternativas } \\
\text { para a sua vida }\end{array}$ & 0,72 & 0,52 \\
\hline 32. São pessoas em quem não se pode confiar & 0,61 & 0,37 \\
\hline
\end{tabular}

Nota. $N=361$. AFC = Análise Fatorial Confirmatória; QARPSSA = Questionário de Atitudes em Relação à População em Situação de Sem-Abrigo.

A análise da fidedignidade do QARPSSA, através do cálculo do alfa de Cronbach, revelou um valor de 0,77. Já no que se refere à $F C$ foi observado um valor de 0,91 . Os valores do alfa de Cronbach para cada uma das dimensões foram os seguintes: 0,84 para a dimensão cognitiva, 0,70 para a dimensão emocional e 0,52 para a dimensão comportamental. Cada uma destas dimensões cognitiva, emocional e comportamental, apresentou uma correlação com o total do QARPSSA de 0,84, 0,70 e 0,48, respetivamente. Relativamente à análise da estabilidade temporal, a realização de um teste $t$ para amostras emparelhadas mostrou a inexistência de diferenças entre os scores do QARPSSA nos dois momentos de avaliação considerados [ $\left.t_{(35)}=0,598 ; p=0,554\right]$. De acrescentar ter-se verificado um valor de $r=0,59, p<0,001$, resultante do cálculo do coeficiente de correlação de Pearson entre os scores destes dois momentos.

Foram também examinadas as associações entre o total do QARPSSA e os demais instrumentos (Tabela 4). 


\section{Tabela 4}

Correlações entre o QARPSSA e os Demais Instrumentos

\begin{tabular}{lccccc}
\hline & IAPSSM & EME & EMI & EDS & EDS20 \\
\hline QARPSSA-T & $0,29^{* *}$ & $0,22^{* *}$ & $-0,06$ & $-0,25^{* *}$ & $-0,03$ \\
\hline
\end{tabular}

Nota. $N=361$. QARPSSA-T = Pontuações totais do Questionário de Atitudes em Relação à População em Situação de Sem-Abrigo; IAPSSM = Inventário de Atitudes face à Procura de Serviços de Saúde Mental; EME = Escala Motivação Externa; EMI = Escala de Motivação Interna; EDS = Escala de Distância Social; EDS-20 = Escala de Desejabilidade Social.

$* * p<0,01$.

O QARPSSA apresentou correlações estatisticamente significativas de efeito pequeno com o IAPSSM, a EME e a EDS, sendo a última uma correlação negativa.

Tendo sido examinadas as correlações entre o QARPSSA e as variáveis sociodemográficas idade e anos de escolaridade, não se verificaram correlações estatisticamente significativas ( $p>0,050)$. Por último, foi explorada a eventual existência de diferenças entre homens e mulheres relativamente ao score total do QARPSSA, não tendo sido encontradas diferenças $\left[t_{(288)}=0,62 ; p=0,534\right]$.

\section{Discussão}

O presente estudo teve como objetivo o desenvolvimento de uma versão exploratória de um questionário de atitudes em relação às pessoas em situação de sem-abrigo, analisando a sua estrutura fatorial e características psicométricas. Atendendo a que se pretendia o alcance de um instrumento breve, foram usados critérios mais restritivos, que conduziram à remoção de 15 itens. De acordo com as recomendações de Hinkin (1995), instrumentos com um número mais reduzido de itens, que mantenham boas propriedades psicométricas, apresentam a vantagem de desencadear menor cansaço nos respondentes ou enviesamento de respostas e, para além disso, a sua administração é menos demorada.

Comparando a distribuição dos itens pelos três fatores com o modelo teórico usado para a construção do questionário, os itens agruparam-se da forma esperada, à exceção do item 21 (Quando vejo uma pessoa em situação de sem-abrigo sinto admiração), que teoricamente foi considerado como pertencente à dimensão emocional e que na ACP saturou na dimensão comportamental. Ainda assim, se for tida em conta a existência de interdependência entre estas dimensões, é possível hipotetizar que um sentimento de admiração pode motivar uma ação, pode levar a fazer algo e, como tal, estar também associado a um potencial comportamento. Convém ainda mencionar que o Item 21 foi precisamente o item que na AFC evidenciou o peso fatorial mais baixo, sendo que em estudos futuros desta medida será conveniente avaliar a sua manutenção neste instrumento.

Tendo sido testado, através de AFC, um modelo de ordem superior composto pelos fatores cognitivo, emocional e comportamental do QARPSSA, observou-se, na globalidade, um bom ajustamento deste modelo, sendo que apenas o indicador de ajustamento do qui-quadrado normalizado evidenciou um valor sofrível (Marôco, 2010). De notar que este indicador é particularmente sensível à dimensão da amostra, pelo que se sugere que estudos futuros sejam conduzidos em amostras de maior dimensão. De acrescentar que surgiu a necessidade de proceder à correlação entre resíduos dos Itens 19 ( $N a$ presença de uma pessoa em situação de sem-abrigo sinto nojo/repulsa) e 20 (Na presença de uma pessoa em situação de sem-abrigo sinto medo de ser contagiado com 
doenças), pertencentes ao mesmo fator, os quais apresentam semelhança ao nível do seu conteúdo. Com efeito, é muitas vezes necessário localizar erros de medida correlacionados entre itens com este tipo de características (Brown, 2003). Quanto aos pesos fatoriais, todos os itens demonstraram pesos elevados, sendo que o mais baixo foi encontrado para o Item 21. De realçar que, apesar da existência das três dimensões atitudinais, Neiva e Mauro (2011) referem que nem sempre é fácil replicar este modelo na análise fatorial o que, por vezes, se constitui como uma crítica ao modelo tripartido. No presente instrumento, esta crítica não se concretizou atendendo a que os resultados da AFC confirmaram a adequação do modelo teórico testado.

Relativamente à consistência interna do score total do QARPSSA, observaram-se valores de alfa de Cronbach e de fiabilidade compósita considerados adequados (Kline, 2000). O mesmo é válido para a consistência interna das dimensões cognitiva e emocional do QARPSSA. Contudo, e ainda que de acordo com Hair et al. (1998), valores abaixo de 0,70 possam ser aceitáveis dada a natureza exploratória deste estudo, a dimensão comportamental apresentou um valor inferior a 0,70 , podendo ser sujeita a um melhoramento em estudos subsequentes. Os resultados psicométricos do QARPSSA foram ainda sugestivos de validade discriminante e de fidedignidade testereteste, não se tendo observado diferenças estatisticamente significativas entre os valores médios do QARPSSA nos dois momentos de avaliação considerados e tendo-se verificado uma correlação de dimensão grande entre os valores destes dois momentos (Cohen et al., 2003).

Ao analisar as correlações do QARPSSA com as demais medidas, foram observadas correlações estatisticamente significativas de pequena dimensão com o IAPSSM, a EME e a EDS, sendo a última uma correlação negativa. Estes resultados, no que concerne à direção da associação, estão de acordo com o era esperado. Tanto o IAPSSM como a EME medem constructos com uma direção positiva, tal como o QARPSSA, enquanto a EDS mede um constructo segundo o qual pontuações mais altas indicam uma maior distância social (isto é, menor proximidade). No que respeita à desejabilidade social não se verificou uma associação com significância estatística com o score total do QARPSSA, o que sugere que os resultados não terão sido influenciados pela desejabilidade social, ainda que a seguir se faça referência a uma limitação a atender futuramente.

\section{Limitações}

Os resultados do presente estudo devem ser lidos tendo em conta algumas limitações. Segundo Gouveia et al. (2009), uma das vantagens da medição de atitudes implícitas é diminuir ou anular o efeito da desejabilidade social, comum nas pesquisas de autorrelato. Neste sentido, tendo em conta que no presente estudo não foi usada uma medida das atitudes implícitas, esta pode ser considerada como uma limitação deste estudo. Apesar do conhecimento da sua importância e do esforço para implementar esse tipo de medição atitudinal, tal não foi exequível, pelo que deverá ser contemplado em futuras investigações.

De referir que o recrutamento dos participantes através das redes sociais e a recolha de dados com recurso a uma plataforma online apresenta também limitações, como o enviesamento da amostra ou a falta de representatividade da população e, consequentemente, restringe a possibilidade de generalizações (Wright, 2005).

Adicionalmente, a condução do estudo exclusivamente em Portugal, faz com que estes resultados não se possam estender à realidade de outros países, pelo que outros estudos com o QARPSSA em outras línguas poderão clarificar este aspeto. 


\section{Conclusão}

Na generalidade, e apesar das limitações mencionadas, o QARPSSA revelou ser um novo instrumento com adequada fidedignidade, que poderá ser usado em vários contextos de forma a aferir as atitudes em relação às pessoas em situação de sem-abrigo. Importa destacar que a avaliação de atitudes pode ser feita através de uma análise quantitativa e qualitativa. Nesta sequência, ao analisar qualitativamente os itens, há a possibilidade de identificar quais são, especificamente, as cognições, emoções e comportamentos que condicionam/influenciam determinado comportamento, o que permite uma intervenção mais ampla e direcionada a nível cognitivocomportamental. Ao conhecer, de forma mais aprofundada, as atitudes da sociedade perante a população em situação de sem-abrigo, considera-se, ainda, que o contributo deste instrumento se pode direcionar para vários contextos:

a) Numa perspetiva educacional e de prevenção (educação para a cidadania) - com a população mais jovem pode possibilitar a criação de conteúdos educacionais adequados, passíveis de serem lecionados.

b) No público em geral, poderá ser útil para o desenvolvimento de campanhas e atividades de sensibilização.

c) Para profissionais do âmbito comunitário, social ou de saúde - os profissionais que trabalham de forma direta com a população em situação de sem-abrigo poderão ter uma visão mais holística e empática acerca deste fenómeno.

Como tal, a versão exploratória do QARPSSA permite, não só uma avaliação das atitudes dos profissionais como, também, a adequação de conteúdos para a sua formação.

Agradecimentos | Acknowledgements: Os autores gostariam de agradecer a todos os participantes pela colaboração.

Conflito de interesses | Conflict of interest: Nenhum | None.

Fontes de financiamento | Funding sources: Nenhuma | None.

Contributos: AC: Pesquisa bibliográfica; Revisão da literatura; Desenvolvimento do Instrumento; Preparação das medidas e escrita do protocolo; Recrutamento dos participantes; Escrita do manuscrito. AG: Desenho do estudo; Preparação das medidas e escrita do protocolo; Análise estatística; Supervisão da escrita do manuscrito e aprovação do manuscrito final. IMC: Revisão e aprovação do manuscrito final. MC: Análise estatística; Revisão e aprovação do manuscrito final.

\section{Referências}

Agans, R. P., \& Liu, G. (2015). Public attitudes toward the homeless. Global Journal of Science Frontier Research, 15(3), 1-8. https://bit.ly/3eOJuR6

Aldeia, J. (2014). Para além do pecado e da doença. Desconstruindo discursos sobre o fenómeno dos sem-abrigo. Revista Portuguesa da Associação Portuguesa de Sociologia 8, 5-26. https://bit.ly/3omdarL

Almiro, P. A., Almeida, D., Ferraz, A. M., Ferreira, R., Perdiz, C., Dias, I. S., Gonçalves, S., Sousa, L. B., \& Simões, M. R. (2017). Escala de Desejabilidade Social de 20 itens (EDS-20). Em M. R. Simões, L. S. Almeida, \& M. M. Gonçalves (Eds.), Psicologia forense: Instrumentos de avaliação (pp. 335-352). Pactor-Lidel.

Bizarrias, F. S., \& Brandão, M. M. (2017). Proposta de modelo conceitual de atitudes implícitas e explícitas no Varejo. Revista Brasileira de Marketing, 16(4), 454-468. https://bit.ly/3uMzPA2

Bogardus, E. S. (1933). A social distance scale. Sociology and Social Research, 17, 265-271. https://bit.ly/33LPmUX

Brown, T. A. (2003). Confirmatory factor analysis of the Penn State Worry Questionnaire: Multiple factors or method effects. Behavior Research and Therapy, 41, 1411-1426. https://doi.org/bvqbvk 
Cohen, J., Cohen, P., West, S. G., \& Aiken, L. S. (2003). Applied multiple regression/correlation analysis for the behavioral sciences ( $3^{\mathrm{a}}$ ed.). Lawrence Erlbaum Associates.

Copeland, D. J., Johnson, P., \& Moore, B. (2020). Effects of a service-learning experience on health-related students' attitudes toward the homeless. Nursing Forum, 56(1), 45-51. https://doi.org/gcmv

Cruz, M. (2018). Opinião pública sobre as pessoas sem-abrigo em Portugal. [Dissertação de mestrado, Universidade do Minho]. RepositóriUM. https://bit.ly/3fpleDf

DeVellis, R. F. (2011). Scale development: Theory and applications (3a ed.). SAGE Publications, Inc.

Eagly, A. H., \& Chaiken, S. (1993). The psychology of attitudes. Harcourt Brace Jovanovich College Publishers. https://bit.ly/3fmPxeB

Fiske, S. T. (2018). Social beings: Core motives in social psychology (4 ${ }^{\mathrm{a}}$ ed., pp. 184-224). John Wiley \& Sons, Inc.

Fonseca, A., Silva, S., \& Canavarro, M. C. (2017). Características psicométricas do Inventário de Atitudes face à Procura de Serviços de Saúde Mental: Estudo em mulheres no período perinatal. Psychologica, 60(2), 65-81. https://doi.org/gcmw

Fornell, C., \& Larcker, D. (1981). Evaluating structural equation models with unobservable variables and measurement error. Journal of Marketing Research, 18(1), 39-50. https://doi.org/cwp

Gouveia, V. V., Guerra, V. M., Souza, D. M. F., Santos, W. S., \& Costa, J. M. (2009). Escala de Desejabilidade Social de Marlowe-Crowne: Evidências de sua validade fatorial e consistência interna. Avaliação Psicológica, 8, 87-98. https://bit.ly/2QjO6VQ

Gouveia, V. V., Mendes, L. A., Freire, S. E., Freires, L. A., \& Barbosa, L. H. (2014). Medindo associação implícita com o freelAT em português: Um exemplo com atitudes implícitas frente ao poliamor. Psicologia Reflexão e Critica, 27(4), 679-688. https://doi.org/gcmx

Greenwald, A. G., \& Banaji, M. R. (1995). Implicit social cognition: Attitudes, self-esteem, and stereotypes. Psychological Review, 102(1), 4-27. https://doi.org/gmt

Greenwald, A. G., McGhee, D. E., \& Schwartz, J. L. K. (1998). Measuring individual differences in implicit cognition: the implicit association test. Journal of Personality and Social Psychology, 74(6), 1464-1480. https://doi.org/c2cjtx

Greenwald, A. G., Banaji, M. R., Rudman, L. A., Farnham, S. D., Nosek, B. A., \& Mellot, D. S. (2002). A unified theory of implicit attitudes, stereotypes, self-esteem, and self-concept. Psychological Review, 109(1), 325. https://doi.org/bvjp8f

Grupo de Trabalho para a Monitorização e Avaliação da ENIPSSA. (2019). Inquérito caracterização das pessoas em situação de sem-abrigo - 31 de dezembro 2019. https://bit.ly/3tOGdWb

Guzewicz, T. D., \& Takooshian, H. (1992). Development of a short-form scale of public attitudes toward homelessness. Journal of Social Distress and the Homeless, 1(1), 67-79. https://doi.org/fsmd42

Hair, J. F., Tatham, R. L., \& Black, W. C. (1998). Multivariate data analysis (5 ed.). Prentice-Hall.

Hinkin, T. R. (1995). A review of scale development practises in the study of organisations. Journal of Management, 21(5), 967-988. https://doi.org/d3j484

Hocking, J. E., \& Lawrence, S. G. (2000). Changing attitudes toward the homeless: The effects of prosocial communication with the homeless. Journal of Social Distress and the Homeless, 9(2), 91-110. https://doi.org/c8sxzr

Klarare, A., Wikman, A., Söderlund, M., McGreevy, J., Mattson, E., \& Roseblad, A. (2020). Translation, cross-cultural adaptation, and psychometric analysis of the Attitudes Towards Homelessness Inventory for use in Sweden. Worldviews on Evidence-Based Nursing, 18(1), 42-49. https://doi.org/gh78m2

Kline, P. (2000). The handbook of psychological testing ( $2^{\mathrm{a}}$ ed.). Routledge.

Krajewska-Kułak, E., Kułak-Bejda, A., Łukaszuk, C., Guzowski, A., Cybulski, M., Stelcer, B., Jasinski, M., \& Kulak, W. (2016). Attitudes of medical students towards homeless people. Problemów Higieny i Epidemiologii, 97(4), 377-381. https://bit.ly/3ePHY11

Lester, H. E., \& Pattison, H. M. (2000). Development and validation of the Attitudes Towards the Homeless Questionnaire. Medical Education, 34(4), 266-268. https://doi.org/dz36n2

Lima, M. L, \& Correia, I. (2013). Atitudes: medida, estrutura e funções. Em J. Vala \& M. B. Monteiro. Psicologia social (9å ed. pp. 201-243). Fundação Calouste Gulbenkian.

Mackenzie, C. S., Knox, V. J., Gekoski, W. L., \& Macaulay, H. L. (2004). An adaptation and extension of the Attitudes Toward Seeking Professional Psychological Help Scale. Journal of Applied Social Psychology, 34(11), 24102433. https://doi.org/bq3526

Marôco, J. (2010). Análise de equações estruturais: Fundamentos teóricos, software \& aplicações. ReportNumber. 
Mather, D. M., Jones, S. W., \& Moats, S. (2017). Improving upon Bogardus: Creating a more sensitive and dynamic Social Distance Scale. Survey Practice, 10(4), 1-9. https://doi.org/gcmz

Miguel, M., Ornelas, J., \& Maroco, J. (2010). Modelo de atitudes face ao sem-abrigo em Portugal. Análise Psicológica, 3(27), 437-450. https://doi.org/10.14417/ap.310

Neiva, E. R., \& Mauro, T. G. (2011). Atitudes e mudanças de atitudes. Em C. V. Torres, \& E. R. Neiva (Eds.). Psicologia social: principais temas e vertentes (pp. 171-203). Artmed.

OECD. (2020). HC3.1 Homeless-population. https://bit.ly/3uQROQR

Palma, T., \& Maroco, J. (2008). Motivação interna e motivação externa para responder sem preconceito: Tradução, adaptação e validação das duas escalas para a população portuguesa. Laboratório de Psicologia, 6(1), 15-24. https://doi.org/gcm2

Petit, J. M., Loubiere, S., Vargas-Moniz, M. J., Tinland, A., Spinnewijn, F., Greenwood, R. M., Santinello, M., Wolf, J. R., Bokszczanin, A., Bernad, R., Kallmen, H., Ornelas, J., \& Auquier, P. (2018). Knowledge, attitudes, and practices about homelessness and willingness-to-pay for housing-first across 8 European countries: A survey protocol. Archives of Public Health, 76, Artigo 71. https://doi.org/ghd9gb

Phillips, L. (2015). Homelessness: Perception of causes and solutions. Journal of Poverty, 19, 1-19. https://doi.org/gcm3

Plant, E. A., \& Devine, P. G. (1998). Internal and external motivation to respond without prejudice. Journal of Personality and Social Psychology, 75(3), 811-832. https://doi.org/b7kztv

Raykov, T. (1997). Estimation of composite reliability for congeneric measures. Applied Psychological Measurement, 21(2), 173-184. https://doi.org/fp4jgg

Resolução do Conselho de Ministros n.ํ 107/2017, de 25 de julho. (2017). Diário da República n.o 142. https://bit.ly/2RWJky2

Snow-Hill, N. L. (2019). The survey of attitudes toward homeless people: The validation of a new instrument assessing negative attitudes toward homeless people. [Doctoral dissertation, University of South Carolina] Scholar Commons. https://bit.ly/3fkxRAp

Somerville, P. (2013). Understanding homelessness. Housing, Theory and Society, 30(4), 384415. https://doi.org/gft2kc

Tabachnick, B. G., \& Fidell, L. S. (2007). Using multivariate statistic (5 ${ }^{\mathrm{a}}$ ed.). Allyn and Bacon.

Tompsett, C. J., Toro, P. A., Guzicki, M., Manrique, M., \& Zatakia, J. (2006). Homelessness in the United States: Assessing changes in prevalence and public opinion, 1993-2001. American Journal of Community Psychology, 37(1-2), 47-61. https://doi.org/fwwvtw

Toro, P. A., \& McDonell, D. M. (1992). Beliefs, attitudes, and knowledge about homelessness: A survey of the general public. American Journal of Community Psychology, 20(1), 53-80. https://doi.org/c39cfk

Toro, P. A., Tompsett, C. J., Lombardo, S., Philippot, P., Nachtergael, H., Schlienz, N., Stammel, N., Yabar, Y., Blume, M., MacKay, L., \& Harvey, K. (2007). Homelessness in Europe and United States: A comparison of prevalence and public opinion. Journal of Social Issues, 63(3), 505-524. https://doi.org/c4c2jz

Tsai, J., Lee, C. Y. S., Shen, J., Southwick, S. M., \& Pietrzak, R. H. (2018). Public exposure and attitudes about homelessness. Journal of Community Psychology, 47(1), 76-92. https://doi.org/gcm4

Wark, C., \& Galliher, J. F. (2007). Emory Bogardus and the origins of the social distance scale. The American Sociologist, 38, 383-395. https://doi.org/cnf6mx

Wisehart, C. G., Whatley, M. A., \& Briihl, D. S. (2013). The effectiveness of the faces of homelessness educational program on student attitudes toward the homeless. North American Journal of Psychology, 15(3), 483-494. https://bit.ly/3ffvi2A

Wright, K. B. (2005). Researching Internet-based populations: Advantages and disadvantages of online survey research, online questionnaire authoring software packages, and web survey services. Journal of Computer-Mediated Communication, 10(3). https://doi.org/d7z5cr

Wright, J. H., Brown, G. K., Thase, M. E., \& Basco, M. R. (2019). Aprendendo a terapia cognitivo-comportamental: um guia ilustrado (2 ed.). Artmed. 